\title{
BMJ Open MAGnesium-oral supplementation to reduce PAin in patients with severe PERipheral arterial occlusive disease: the MAG-PAPER randomised clinical trial protocol
}

\author{
Monica Aida Venturini, ${ }^{1}$ Sergio Zappa, ${ }^{1}$ Cosetta Minelli, ${ }^{2}$ Stefano Bonardelli, ${ }^{3,4}$ \\ Laura Lamberti, ${ }^{5}$ Luca Bisighini, ${ }^{5}$ Marta Zangrandi, ${ }^{5}$ Maddalena Turin, ${ }^{5}$ \\ Francesco Rizzo, ${ }^{5}$ Andrea Rizzolo, ${ }^{5}$ Nicola Latronico ${ }^{1,5}$
}

To cite: Venturini MA, Zappa S, Minelli C, et al. MAGnesium-oral supplementation to reduce PAin in patients with severe PERipheral arterial occlusive disease: the MAG-PAPER randomised clinical trial protocol. BMJ Open 2015;5: e009137. doi:10.1136/ bmjopen-2015-009137

- Prepublication history for this paper is available online. To view these files please visit the journal online (http://dx.doi.org/10.1136/ bmjopen-2015-009137).

Received 15 July 2015 Revised 16 November 2015 Accepted 17 November 2015

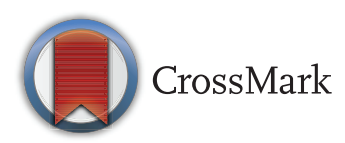

For numbered affiliations see end of article.

Correspondence to Professor Nicola Latronico; nicola.latronico@unibs.it

\section{ABSTRACT}

Introduction: Magnesium exerts analgaesic effects in several animal pain models, as well as in patients affected by acute postoperative pain and neuropathic chronic pain. There is no evidence that magnesium can modulate pain in patients with peripheral arterial occlusive disease (PAOD). We describe the protocol of a single-centre randomised double-blind clinical trial aimed at assessing the efficacy of oral magnesium supplementation in controlling severe pain in patients with advanced PAOD.

Methods and analysis: Adult patients affected by PAOD at stages III and IV of Lèriche-Fontaine classification, who are opioid-naive, and who have been admitted to our Acute Pain Service for intractable pain, will be eligible. Patients will be randomised to the control group, treated with standard therapy (oxycodone and pregabalin) plus placebo for 2 weeks, or to the experimental group (standard therapy plus magnesium oxide). Patients will be evaluated on days $0,2,4,6,8,12$ and 14; the following information will being collected: daily oxycodone dose; average and maximum pain (Numerical Rating Scale); pain relief (Pain Relief Scale); characteristics of the pain (Neuropathic Pain Scale); impact of pain on the patient's daily activities (Brief Pain Inventory). The primary outcome will be oxycodone dosage needed to achieve satisfactory analgaesia on day 14 . Secondary outcomes will be pain relief on day 2, time needed to achieve satisfactory analgaesia and time needed to achieve a pain reduction of $50 \%$. A sample size calculation was performed for the primary outcome, which estimated a required sample size of 150 patients (75 per group).

Ethics and dissemination: Ethical approval of the study protocol has been obtained from Comitato Etico Provinciale di Brescia, Brescia, Italy. Trial results will be disseminated through scientific journal manuscripts and scientific conference presentations.

Trial registration number: NCT02455726.

\section{Strengths and limitations of this study}

- There are no randomised clinical trials on the use of magnesium to control pain in peripheral arterial occlusive disease.

- Magnesium oxide is a low-cost oral drug with high safety profile. Its adoption would also be affordable in low-income and middle-income countries for use in polypharmacological pain therapy.

- As a single-centre trial, generalisability of results may be limited, and will require replication at other centres with different patient populations.

\section{INTRODUCTION}

Background

Magnesium is a physiological voltagedependent blocker of $N$-methyl-D-aspartate (NMDA)-coupled channels. ${ }^{1}{ }^{2}$ Magnesium ions can influence inflammatory pain and neuropathic pain through several different mechanisms, ${ }^{3-6}$ including a decrease in NMDA receptor activity in the central nervous system by blocking the receptorcoupled calcium channel, a reduction in activity of other presynaptic and postsynaptic calcium channels, a reduction of substance $p$ synthesis, a potentiation of the action of morphine in the presynaptic area of dorsal horn, a modulation of release and action of the glutamate, the substance $p$ and the calcitonine gene-related peptide (CGRP) in the spinal cord, and a reduction in thromboxane A2, and other proinflammatory eicosanoids and cytokines outside the central nervous system. $^{78}$

Magnesium has been shown to exert an analgaesic effect both in animals, in particular in rats with diabetic peripheral 
neuropathy, ${ }^{9-11}$ drug-induced neuropathy ${ }^{12}$ and spinal nerve ligation, ${ }^{11} 13$ as well as in humans with a variety of acute and chronic conditions. There is evidence for a positive interactive effect on pain using magnesium with narcotic analgaesics and intravenous general anaesthetics, ${ }^{14-16}$ local anaesthetics ${ }^{15}$ and antidepressants. ${ }^{17} 18$

In humans, magnesium can be considered as an effective adjunct for postoperative analgaesia in the setting of conventional opioid-based therapy: perioperative magnesium can provide a clinically important reduction in opioid consumption in the first $24 \mathrm{~h}$ postoperatively, without any reported serious adverse effects. ${ }^{19}$ Magnesium bolus followed by continuous intravenous infusion leads to significant reduction in both intraoperative dosage of propofol and atracurium, and postoperative morphine consumption. ${ }^{20}$ Magnesium sulfate during total intravenous anaesthesia improves the quality of postoperative analgaesia ${ }^{21} 22$ and prevents remifentanil-induced hyperalgaesia. ${ }^{23}$ Magnesium enhances the analgaesic effect of metamizol on posttonsillectomy pain ${ }^{24}$ and, if administered preoperatively, it is effective in reducing the incidence and severity of postoperative sore throat in the immediate postoperative period. $^{25}$

Magnesium supplementation is effective in neuropathic pain, ${ }^{26-29}$ and can reduce pain intensity and improve lumbar spine mobility in patients with refractory chronic low back pain with a neuropathic component. ${ }^{30}$

However, findings on the efficacy of magnesium on pain reduction are not consistent across studies. ${ }^{31}$ Pickering $e t a l^{22}$ showed that magnesium alone did not improve neuropathic pain compared to placebo. Felsby $e t a l^{33}$ failed to demonstrate any reduction in pain following magnesium administration in patients with chronic neuropathic pain. Mikkelsen $e$ t $a l^{34}$ tested the effect of magnesium sulfate administration on hyperalgaesia following heat/capsaicin stimulation in healthy volunteers and found no evidence of benefit.

In patients with advanced-stage peripheral arterial occlusive disease (PAOD), pain is severe and is a major factor in limiting patients' activities in daily life. The characteristics of ischaemic pain change from nociceptive pain in patients with intermittent claudication to predominantly neuropathic pain in patients with critical limb ischaemia. ${ }^{35}$ Oral magnesium supplementation improves endothelial function in patients with systemic arterial disease and improves cardiac exercise tolerance and quality of life in patients with coronary artery disease. ${ }^{36}$ Magnesium supplementation has therefore a preventive role in atherosclerosis, and may exert an analgaesic effect on vascular pain.

There are no randomised controlled studies evaluating the efficacy of oral magnesium as a supplemental treatment in patients with PAOD-related pain.

\section{Objectives and hypotheses}

Our aim is to study the effect of oral magnesium supplementation in opioid-naive patients with PAOD with stage
III or IV disease according to Lèriche-Fontaine classification, ${ }^{37}$ and severe acute pain.

The hypothesis is that oral magnesium can contribute to achieving a good level of analgaesia while reducing opioid dosage in an elderly population affected by atherosclerosis.

\section{METHODS}

\section{Trial design and study setting}

This is a single-centre randomised double-blind clinical trial conducted at the Department of Anaesthesia, Critical Care Medicine and Emergency and the Department of General Surgery of the Spedali Civili, a large, regional hospital affiliated with the University of Brescia and serving a population of 1 million people in the north-east of Italy. All consecutive patients with PAOD attending the inpatient Acute Pain Service will be evaluated by the anaesthesiologist in charge and invited to take part in the study, if eligible.

The protocol adheres to the SPIRIT standards (http://www.spirit-statement.org/).

\section{Participants}

The study will recruit all adult patients (aged 18 years or above) presenting with pain due to PAOD at stages III and IV according to the Lèriche-Fontaine classification, and with no treatment with opioids and magnesium at the time of recruitment.

Patients will be excluded if they present any of the following conditions: renal failure (serum creatinine $\geq 2.0 \mathrm{mg} / \mathrm{dL}$ ); congestive heart failure (New York Heart Association, NYHA >3); treatment with digoxin and/or calcium-channel blockers; pre-existing neuromuscular diseases; chronic diarrhoea; acute limb ischaemia successfully treated with interventional angiography; Fogarty embolectomy and/or surgical revascularisation.

\section{Intervention, procedures and standard care}

Patients will be evaluated using the Numeric Rating Scale (NRS), the Pain Relief Scale (PRS), the Neuropathic Pain Scale (NPS) and the Brief Pain Inventory (BPI).

NRS is designed to evaluate average and maximum pain intensity with a score of 0 indicating no pain and a score of 10 indicating the worst pain, while $\mathrm{PRS}^{38}$ evaluates pain relief, ranging from $0 \%$ indicating no pain relief to $100 \%$ indicating complete pain relief. At shortterm evaluation, the assessment of pain relief is more appropriate than assessment of pain intensity, because all patients have the same baseline score (0 pain relief), whereas pain intensity scores at baseline can be different. NPS ${ }^{39-42}$ describes the characteristics of neuropathic pain and consists of 10 items characterising patient's pain in terms of reactions to light touch or clothing.

Finally, $\mathrm{BPI}^{41}{ }^{42}$ is designed to assess the impact of pain on patients' activities in daily life on admission (day $0)$ and on day 14 . 
Patients with PAOD at stages III and IV have severe pain at rest and the use of opioids represents a standard of care. Therefore, magnesium will be used as supplemental treatment. Eligible patients will be randomised to standard therapy plus magnesium oxide $300 \mathrm{mg}$ twice a day or standard therapy with placebo, consisting of fructose $10 \mathrm{~g}$. We will use an oral soluble preparation of magnesium (Mag Orosolubile, Sanofi Aventis), one dose of which contains magnesium oxide $300 \mathrm{mg}$, citric acid, monosodium citrate, sorbitol, aspartame, sodium cyclamate and lemon flavouring.

Standard therapy consists of an oral administration of oxycodone $5 \mathrm{mg}$ and pregabalin $25 \mathrm{mg}$ /day for 2 weeks. Patients will receive oxycodone hydrochloride $5 \mathrm{mg}$ (Oxycontin, Mundipharma Pharmaceutical) at 8:00 and pregabalin $25 \mathrm{mg}$ (Lyrica, Pfizer Italia Srl) at 20:00. Starting by day 2, the opioid dose will be titrated every $48 \mathrm{~h}$ in order to reach the maximum therapeutic goal and to minimise side effects. At each clinical evaluation on day $2,4,6,8,12$ and 14 , the dose of the oxycodone will be increased by $5-10 \mathrm{mg}$, according to patient's age, body weight, and renal and liver function, to reach a $\mathrm{NRS} \leq 4$. A rescue dose of paracetamol $1 \mathrm{~g}$ will be provided if needed (maximum dose $3 \mathrm{~g} /$ day).

\section{Outcome measure}

\section{Primary outcome measure}

The primary outcome is the daily oxycodone dosage needed to achieve 'satisfactory analgaesia', defined as $\mathrm{NRS} \leq 4$ after 14 days of therapy.

\section{Secondary outcome measures}

The secondary outcomes are: level of pain relief on day 2 measured using PRS; time needed to achieve satisfactory analgaesia $(\mathrm{NRS} \leq 4)$; time needed to achieve a reduction of pain of $50 \%(\mathrm{PRS} \geq 50 \%)$.

\section{Randomisation and blinding}

Participants will be randomly assigned to either the experimental or the control group with a 1:1 allocation ratio using a computer-generated randomisation schedule based on permuted blocks. To ensure allocation concealment, the block size will not be disclosed and allocation will be assigned using sequentially numbered, opaque, sealed envelopes. Trial participants, care providers and data collectors, including outcome assessors and data analysts, will be blinded to group allocation. Magnesium and placebo are white powder, indistinguishable to the eye and to the touch. Oral magnesium and placebo will be prepared by two physicians in the Acute Pain Service using identical test tubes. In particular, the contents of each magnesium sachet will be poured into a small plastic test tube, and the same will be done for the $10 \mathrm{~g}$ of fructose ( 1 measuring spoon). The two physicians will monitor the blinding procedure, but they will not be involved in outcome assessment, data collection and analysis.

\section{Statistical methods}

\section{Sample size calculation}

A sample size calculation was performed for the primary outcome, that is, oxycodone dosage needed to achieve a $\mathrm{NRS} \leq 4$ on day 14 . We considered a mean oxycodone dose of $20.25 \mathrm{mg}$ (SD $10 \mathrm{mg}$ ) on day 14 in the control group, based on data from 270 patients with advanced PAOD treated by our Acute Pain Service in the period 2012-2014. For a significance threshold of $5 \%(p<0.05$, two-sided test), 116 patients (58 per group) would be needed to achieve $90 \%$ power to detect a minimum reduction in oxycodone dose of $30 \%$ in the experimental compared with the control group (ie, mean oxycodone dose of $14.18 \mathrm{mg}$ in the experimental group). The target sample size was increased to 150 patients ( 75 per group) to account for a possible dropout rate of approximately $20 \%$. The sample size of 150 patients allows for an $80 \%$ power to detect an increase in pain relief on day 2 (secondary outcome) from $10 \%$ in the control group to a minimum of $30 \%$ in the experimental group.

\section{Statistical analyses}

Descriptive statistics will be used to summarise patients' characteristics and baseline outcome data in the two treatment groups. Differences between experimental and control group in daily oxycodone dose on day 14 (primary outcome) and pain relief on day 2 (secondary outcome) will be analysed using a t test if the data (original or transformed) are normally distributed, and with a Mann-Whitney $\mathrm{U}$ test if normality cannot be achieved. For the two time-to-event secondary outcomes (time needed to achieve satisfactory analgaesia and $50 \%$ pain reduction), differences between the two groups will be tested using a log-rank test. In the case of imbalance between the two groups in baseline characteristics strongly associated with the outcome (confounding by chance), sensitivity analyses with adjustment for these covariates will be performed, using multiple linear regression and Cox regression. Analyses will be performed according to the intention to treat principle.

All tests will be two tailed, and $\mathrm{p}<0.05$ will be considered as significant. For binary outcomes, ORs will be estimated with their 95\% CI. Analyses will be performed using STATA V.13.0 (Stata Corp, College Station, Texas, USA) software.

\section{RESULTS}

\section{Participant flow diagram}

Figure 1 shows the study flow reported according to the Consolidated Standards of Reporting Trials (CONSORT).

\section{Recruitment time frame}

Patients meeting the inclusion criteria will be invited to participate, with written informed consent obtained before randomisation. Patients will be randomised as 
Figure 1 Study flow will be reported according to the Consolidated Standards of Reporting Trials (CONSORT).

\section{Assessed for eligibility $(n=\ldots)$}

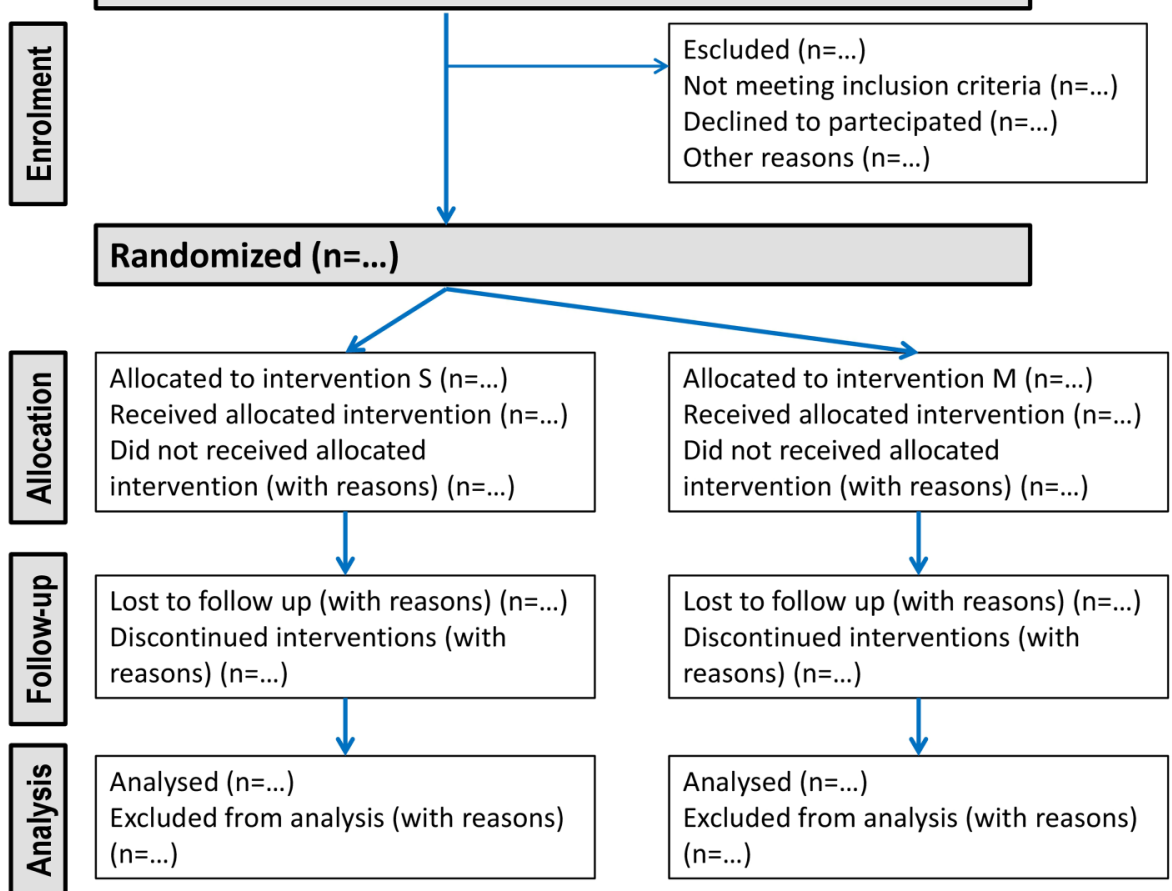

soon as they need the first dose of opioid for pain management, as assessed by the Acute Pain Service team, and the treatment will last 14 days. Patients discharged from the hospital before day 14 will be discharged with the pain therapy (including magnesium or placebo) and will be assessed as outpatients in our ambulatory pain clinic at the expected intervals.

\section{Data collection}

The following patient characteristics will be recorded at baseline (table 1): age, gender, body weight and height, lifestyle habits (including smoking) and comorbidities

\begin{tabular}{|c|c|c|c|c|c|c|c|}
\hline \multirow[b]{2}{*}{ Data collection } & \multicolumn{7}{|c|}{ Days } \\
\hline & $\overline{0}$ & 2 & 4 & 6 & 8 & 12 & 14 \\
\hline Age & $x$ & & & & & & \\
\hline Gender & $x$ & & & & & & \\
\hline Weight/height & $x$ & & & & & & \\
\hline Smoke & $x$ & & & & & & \\
\hline Diabetes & $x$ & & & & & & \\
\hline Cardiac events & $x$ & & & & & & \\
\hline Cerebrovascular events & $x$ & & & & & & \\
\hline Oxycodone dose, mg & & $x$ & $x$ & $x$ & $\mathrm{x}$ & $x$ & $x$ \\
\hline Rescue dose paracetamol & & $x$ & $x$ & $x$ & $x$ & $x$ & $x$ \\
\hline Average pain, NRS & $x$ & $x$ & $x$ & $x$ & $x$ & $x$ & $x$ \\
\hline Maximum pain, NRS & $x$ & $x$ & $x$ & $x$ & $x$ & $x$ & $x$ \\
\hline Pain relief & & $x$ & $x$ & $x$ & $x$ & $x$ & $x$ \\
\hline Neuropathic Pain Scale & $x$ & $x$ & $x$ & $x$ & $x$ & $x$ & $x$ \\
\hline Brief Pain Inventory & $x$ & & & & & & $x$ \\
\hline Side effects & & $x$ & $x$ & $x$ & $x$ & $x$ & $x$ \\
\hline
\end{tabular}

(diabetes, cardiac and cerebrovascular events, and cancer, as well as Charlson Comorbidity Index ${ }^{43} 44$ ).

The following data on pain will be recorded on day 0 (baseline): patient's perceived average and maximum pain (NRS); pain characteristics (NPS); impact of pain on patient's daily activities (BPI) (table 1 ).

The following data on pain and treatment will be recorded on day 2, 4, 6, 8, 12 and 14: daily oxycodone dose; number of paracetamol rescue doses requested and administered; patient's perceived average and maximum pain (NRS); pain relief (PRS); pain characteristics (NPS); gastrointestinal side effects, including diarrhoea/constipation. Impact of pain on patient's daily activities will be recorded on day 14 (table 1 ).

\section{DISCUSSION}

Interpretation, limitations and generalisability

Opioid analgaesics are risky drugs in patients with chronic non-cancer pain; they can be effective in treating a variety of chronic pain conditions, but their use can be potentially accompanied by side effects and complications: not only abuse, addiction and tolerance, but also sedation, dizziness, drowsiness, constipation, nausea, vomiting and delayed gastric emptying, etc. ${ }^{45}$

These side effects are very crippling for old patients, who may lose their self-reliance. Magnesium may aid in maximising the effectiveness of opioids while reducing their dose, and thus the severity of side effects.

In addition to its NMDA-blocking properties, magnesium has vasodilatory effects, improves endothelial function $^{36}$ and exerts antidepressant action. ${ }^{11}$ If proved effective, the analgaesic effect might be due to a combined mechanism. Magnesium has a high safety profile, 
lacks serious side effects and is a low cost drug. The most frequent and expected side effect is mild diarrhoea, which could be helpful in counteracting opioid-related constipation.

As a single-centre RCT, demonstration of the analgaesic efficacy of magnesium supplementation would require replication in other centres before magnesium is adopted as a standard of treatment in advanced-stage patients with PAOD with severe pain; in particular, generalisability of results to patients with PAOD with less severe disease or to other conditions characterised by acute neuropathic pain would require further evidence.

\section{ETHICS AND DISSEMINATION}

Detailed written information will be provided to the patients about the study protocol, the purpose of research and the safety of magnesium treatment. Written informed consent will be requested from all patients before randomisation. All investigations will be conducted according to the principles expressed in the Declaration of Helsinki.

All study-related information will be stored securely at the study site; sensible information will be stored in locked file cabinets in areas with limited access. All records that contain names or other personal identifiers will be stored separately from study records identified by code number. Participants' study information will not be released outside of the study without the written permission of the participant.

Trial results will be disseminated through scientific journal manuscripts and scientific conference presentations.

\section{Author affiliations}

${ }^{1}$ Department of Anesthesia, Critical Care Medicine and Emergency, University of Brescia at Spedali Civili, Brescia, Italy

${ }^{2}$ National Heart and Lung Institute, Imperial College London, London, UK

${ }^{3}$ Department of Surgery, University of Brescia at Spedali Civili, Brescia, Italy

${ }^{4}$ Department of Clinical and Experimental Sciences, University of Brescia, Brescia, Italy

${ }^{5}$ Department of Medical and Surgical Specialties, Radiological Sciences and Public Health, University of Brescia, Brescia, Italy

Twitter Follow Andrea Rizzolo at @Andrearizzolo and NICOLA LATRONICO at @NI1956

Contributors MAV and SZ conceived of the study. MAV, SZ, SB and NL planned the study. LL, LB, MZ, MT, FR and AR will be responsible for data collection, quality analysis and storage. CM provided statistical expertise in clinical trial design and will conduct the statistical analysis. All the authors contributed to refinement of the study protocol and approved the final manuscript.

Funding The study is funded by the Department of Medical and Surgical Specialties, Radiological Sciences and Public Health of the University of Brescia, Brescia, Italy. The management of patients, and analysis and reporting of the study, will be conducted independently by the study investigators.

Competing interests None declared.

Patient consent Obtained.

Ethics approval Comitato Etico Provinciale di Brescia, protocol number 0036604, 13/06/2014.
Provenance and peer review Not commissioned; externally peer reviewed.

Open Access This is an Open Access article distributed in accordance with the Creative Commons Attribution Non Commercial (CC BY-NC 4.0) license, which permits others to distribute, remix, adapt, build upon this work noncommercially, and license their derivative works on different terms, provided the original work is properly cited and the use is non-commercial. See: http:// creativecommons.org/licenses/by-nc/4.0/

\section{REFERENCES}

1. Iseri LT, French JH. Magnesium: nature's physiologic calcium blocker. Am Heart J 1984;108:188-93.

2. Kew JN, Kemp JA. Ionotropic and metabotropic glutamate receptor structure and pharmacology. Psycopharmacology 2005;179:4-29.

3. Recio-Pinto E, Castillo C. Peripheral N-methyl-D-aspartate receptors as possible targets for chronic pain treatment. Techn Reg Anesth Pain Manag 2010;14:48-58.

4. Fisher K, Coderre TJ, Hagen NA. Targeting the N-Methyl-D-Aspartate receptor for chronic pain management: preclinical animal studies, recent clinical experience and future research directions. J Pain Symptom Manage 2000;20:358-73.

5. Hewitt DJ. The use of NMDA-receptor antagonists in the treatment of chronic pain. Clin J Pain 2000;16:S73-9.

6. De Kock MF, Lavand'homme PM. The clinical role of NMDA receptor antagonists for the treatment of postoperative pain. Best Pract Res Clin Anaesthesiol 2007;21:85-98.

7. Petrenko AB, Yakamura T, Baba H, et al. The role of N-Methyl-D Aspartate NMDA receptors in pain: a review. Anesth Analg 2003;97:1108-16.

8. Nechifor M. Magnesium involvement in pain. Magnes Res 2011;24:220-2.

9. Hasanein P, Parviz M, Keshavarz M, et al. Oral magnesium administration prevents thermal hyperalgesia induced by diabetes in rats. Diabetes Res Clin Pract 2006;73:17-22.

10. Rondón LJ, Privat AM, Daulhac L, et al. Magnesium attenuates chronic hypersensitivity and spinal cord NMDA receptor phosphorylation in a rat model of diabetic neuropathic pain. $J$ Physiol 2010;588(Pt 21):4205-15.

11. Begon S, Pickering G, ESchalier A, et al. Magnesium increases morphine analgesic effect in different experimental models of pain. Anesthesiology 2002;96:627-32.

12. Bujalska M, Makulska-Nowak H, Gumulka SW. Magnesium ions and opioid agonists in vincristine-induced neuropathy. Pharmacol Rep 2009;61:1096-104.

13. Ulugol A, Aslantas A, Ipci $Y$, et al. Combined systemic administration of morphine and magnesium sulfate attenuates pain-related behavior in mononeuropathic rats. Brain Res 2002;943:101-4.

14. Chen $\mathrm{Y}$, Zhang $\mathrm{Y}$, Zhu YL, et al. Efficacy and safety of an intra-operative intra-articular magnesium/ropivacaine injection for pain control following total knee arthroplasty. J Int Med Res 2009;37:1733-41.

15. Bondok RS, Abd El-Hady AM. Intra-articular magnesium is effective for postoperative analgesia in arthroscopic knee surgery. $\mathrm{Br} J$ Anaesth 2006;97:389-92.

16. Cizmeci P, Ozkose Z. Magnesium sulphate as an adjuvant to total intravenous anesthesia in septorhinoplasty: a randomized controlled study. Aesth Plast Surg 2007;31:167-73.

17. Nechifor M. Magnesium in major depression. Magnes Res 2009;22: S163-6.

18. Murck H. Ketamine, magnesium and major depression-from pharmacology to pathophysiology and back. J Psychiatr Res 2013;47:955-65.

19. Albrecht E, Kirkham KR, Liu SS, et al. Peri-operative intravenous administration of magnesium sulphate and postoperative pain: a meta-analysis. Anaesthesia 2013;68:79-90.

20. Seyhan TO, Tugrul M, Sungur MO, et al. Effects of three different dose regimens of magnesium on propofol requirements haemodynamic variables and postoperative pain relief in gynaecological surgery. Br J Anaesth 2006;96:247-52.

21. Ryu JH, Kang MH, Park KS, et al. Effects of magnesium sulphate on intraoperative anaesthetic requirements and postoperative analgesia in gynaecology patients receiving total intravenous anaesthesia. Br J Anaesth 2008;100:397-403.

22. Tramer MR, Schneider J, Marti RA, et al. Role of magnesium sulfate in postoperative analgesia. Anesthesiology 1996;84:340-7.

23. Song JW, Lee YW, Yoon KB, et al. Magnesium sulfate prevents remifentanil-induced postoperative hyperalgesia in patients undergoing thyroidectomy. Anesth Analg 2011;113:390-7. 
24. Tugrul S, Degirmenci N, Eren SB, et al. Analgesic effect of magnesium in post-tonsillectomy patients: a prospective randomised clinical trial. Eur Arch Otorhinolatyngol 2015;272:2483-7.

25. Borazan $\mathrm{H}$, Kececioglu A, Okesli S, et al. Oral magnesium lozenge reduces postoperative sore throat: a randomized, prospective, placebo-controlled study. Anesthesiology 2012;117:512-18.

26. Brill S, Sedgwick PM, Hamann W, et al. Efficacy of intravenous magnesium in neuropathic pain. $\mathrm{Br} J$ Anaesth 2002;89:711-14.

27. Crosby V, Wilcock A, Corcoran R. The safety and efficacy of a single dose $(500 \mathrm{mg}$ or $1 \mathrm{~g})$ of intravenous magnesium sulfate in neuropathic pain poorly responsive to strong opioid analgesics in patients with cancer. J Pain Symptom Manage 2000;19:35-9.

28. Suresh S, Lozono S, Hall SC. Large-dose intravenous methotrexate-induced cutaneous toxicity: can oral magnesium oxide reduce pain? Anesth Analg 2003;96:1413-14.

29. Kroin JS, Mc Carthy RJ, Von Roenn N, et al. Magnesium sulfate potentiates morphine antinociceptoin at the spinal level. Anesth Analg 2000;90:913-17.

30. Yousef AA, Al-deeb AE. A double-blinded randomized controlled study of the value of sequential intravenous and oral magnesium therapy in patients with chronic low back pain with a neuropathic component. Anaesthesia 2013;68:260-6.

31. Lysakowski C, Dumont L, Czarnetzki C, et al. Magnesium as an adiuvant to postoperative analgesia: a systematic review of randomized trials. Anesth Analg 2007;104:1532-9.

32. Pickering G, Morel V, Simen E, et al. Oral magnesium treatment in patients with neuropathic pain: a randomized clinical trial. Magnes Res 2011;24:28-35.

33. Felsby S, Nielsen J, Arendt-Nielsen L, et al. NMDA receptor blockade in chronic neurophatic pain: a comparison of ketamine and magnesium chloride. Pain 1996;64:283-91.

34. Mikkelsen S, Dirks J, Fabricius $P$, et al. Effect of intravenous magnesium on pain and secondary hyperalgesia associated with the heat/capsaicin sensitization model in healthy volunteers. $\mathrm{Br} \mathrm{J}$ Anaesth 2001;86:871-3.

35. Rüger LJ, Irnich D, Abahji TN, et al. Characteristics of chronic ischemic pain in patients with peripheral arterial disease. Pain 2008;139:201-8.

36. Shechter M, Merz NB, Stuehlinger HG, et al. Effects of oral magnesium therapy on exercise tolerance, exercise-induced chest pain and quality of life in patients with coronary artery disease. $A m J$ Cardiol 2003;91:517-21.

37. Fontaine R, Kieny R, Gangloff JM, et al. Long-term results of restorative arterial surgery in obstructive diseases of the arteries. J Cardiovasc Surg 1964;5:463-72.

38. Caraceni A, Cherny N, Fainsinger R, et al. Pain measurement tools and methods in clinical research in palliative care: recommendations of an expert working group of the European Association of Palliative Care. J Pain and Symptom Manage 2002;23:239-55.

39. Frampton CL, Hughes-Webb P. The measurement of pain. Clin Oncol 2011;23:381-6.

40. Galer BS, Jensen MP. Development and preliminary validation of a pain measure specific to neuropathic pain: the Neuropathic Pain Scale. Neurology 1997;48:332-8.

41. Bonezzi C, Bettaglio R, Jurusich D, et al. Scala del Dolore Neuropatico, versione italiana di The Neuropathic Pain Scale. Minerva Anestesiol 2000;66:841-3.

42. Negri $\mathrm{E}$, Bettaglio R, Demartini $\mathrm{L}$, et al. Validation if Italian version of the Neuropathic Pain Scale and its clinical implications. Minerva Anestesiol 2002;68:95-104

43. Charlson ME, Pompei P, Ales KL, et al. A new method of classifying prognostic comorbidity in longitudinal study: development and validation. J Chron Dis 1987;40:373-83.

44. Charlson ME, Szatrowski TP, Peterson J, et al. Validation of a combined comorbidity index. J Clin Epidemiol 1994;47:1245-51.

45. Benyamin R, Trescot AM, Datta S, et al. Opioid complications and side effects. Pain Physician 2008;11:S105-20. 